\title{
Thoughts on holographic complexity and its basis dependence
}

\author{
Koji Hashimoto, ${ }^{*}$ Norihiro Iizuka, ${ }^{\dagger}$ and Sotaro Sugishita ${ }^{\ddagger}$ \\ Department of Physics, Osaka University, Toyonaka, Osaka 560-0043, JAPAN
}

(Received 21 May 2018; published 6 August 2018)

\begin{abstract}
In this paper, we argue that holographic complexity should be a basis-dependent quantity. Computational complexity of a state is defined as a minimum number of gates required to obtain that state from the reference state. Due to this minimality, it satisfies the triangle inequality and can be regarded as a (discrete version of) distance in the Hilbert space. However, we show a no-go theorem that any basis-independent distance cannot reproduce the behavior of the holographic complexity. Therefore, if holographic complexity is dual to a distance in the Hilbert space, it should be basis dependent; i.e., it is not invariant under a change of the basis of the Hilbert space.
\end{abstract}

DOI: 10.1103/PhysRevD.98.046002

\section{INTRODUCTION}

What is the boundary dual of the black hole interior in AdS/CFT? If such a quantity exists, it should enable us to probe behind the black hole horizon in terms of the boundary theory. Therefore, it is very interesting to study any candidates for that. In particular, since the interior of a black hole grows linearly in time for a very long period $t \sim e^{S}$ where $S$ is the entropy of the system [1,2] (see also [3] for earlier observation of the late time behavior of the entanglement entropy), the dual quantity should also satisfy this property. In fact, it is conjectured that the dual quantity is quantum computational complexity [1,2], since the maximal complexity for a quantum system seems as big as $e^{S}$, and also complexity, especially that of quantum circuit models, grows linearly in time [1,4]. More concretely, there are two conjectures according to these expectations: the complexity $=$ volume $(\mathrm{CV})$ conjecture $[1,5]$ and the complexity $=$ action $(\mathrm{CA})$ conjecture $[6,7]$. For eternal two-sided black holes, the $\mathrm{CV}$ conjecture states that the complexity is dual to the volume of the maximal time slice anchored at the two given boundary times. The CA conjecture instead proposes that the complexity equals the gravitational action on a region so-called the WheelerDeWitt (WDW) patch. Although the time dependence of the maximal volume and the WDW action are quantitatively different (see [8]), they show the same behavior at

\footnotetext{
*koji@phys.sci.osaka-u.ac.jp †iizuka@phys.sci.osaka-u.ac.jp

*sugishita@het.phys.sci.osaka-u.ac.jp
}

Published by the American Physical Society under the terms of the Creative Commons Attribution 4.0 International license. Further distribution of this work must maintain attribution to the author(s) and the published article's title, journal citation, and DOI. Funded by SCOAP. late times; they grow linearly in time and saturate Lloyd's bound. ${ }^{1}$ Here we call all these 'bulk-defined-quantities' holographic complexity, and denote it by $C_{\mathrm{hol}}$. Here the point is that the late time behavior of holographic complexity is universal, even though their behaviors except at late times depend on the details of the definition.

Recently, there has been many works on the holographic complexity [9-27]. In order to verify the conjectures, we need to know the properties of complexity in quantum field theories (QFTs). However, currently, even a proper definition of it is beyond our reach. Recently there were several proposals for the definition of the complexity for QFTs [28-36], with which the complexity is evaluated and compared with the holographic counterpart. While each argument is concrete, the universal way to define the complexity in QFTs is missing. ${ }^{2}$

Thus, a universal requirement for the complexity in QFTs is called for, and in this paper we attempt to characterize the complexity in QFTs in regard to the properties of the holographic complexity. As we will see soon, complexity is a kind of distance between quantum states. In fact, a geometric approach is proposed to define a complexity in $[37,38]$, and the relation to the holographic complexity was discussed in $[39,40]$ (see also $[32,33,41,42]$ for attempts to define complexities for QFTs as geometric distances). Since there is a variety of distance measures, we need to discriminate proper ones for our purpose. In quantum information, popular definitions of the distance are basis independent.

\footnotetext{
${ }^{1}$ If the growth rate is fractional of the value of Lloyd's bound, one can easily define the holographic complexity by multiplying an overall factor to saturate the bound.

${ }^{2}$ In two dimensions, there is a nice definition of the complexity by the path-integral optimization procedure [28-30]. It is interesting to investigate if one can generalize this to higher dimensions.
} 
We argue that a distance corresponding to the holographic complexity should be a basis-dependent quantity; i.e., it is not invariant under a change of the basis of the Hilbert space. In other words, we show that any basis-independent distance cannot be dual to the holographic complexity.

\section{COMPLEXITY AS A DISTANCE}

Quantum computational complexity (or gate complexity) is a quantity characterizing the difficulty to construct a state from a given reference state. Given a set $\left\{G_{\alpha}\right\}$ of elementary unitary operators called gates $G_{\alpha}$, the complexity $C\left(\psi, \psi_{0}\right)$ of a state $|\psi\rangle$ is defined as the minimum number of gates needed to construct $|\psi\rangle$ from a reference state $\left|\psi_{0}\right\rangle$. Thus, complexity $C\left(\psi, \psi_{0}\right)$ represents how $|\psi\rangle$ is far from $\left|\psi_{0}\right\rangle$ measured with a given gate set. Actually, as we will see as follows, complexity has properties of a distance between two quantum states $|\psi\rangle$ and $\left|\psi_{0}\right\rangle$.

Let us recall the axioms of distance. For a general distance $D(\rho, \sigma)$ between two density matrices $\rho$ and $\sigma{ }^{3}$ it satisfies the following axioms:

$$
\begin{gathered}
D(\rho, \sigma) \geq 0 . \\
D(\rho, \sigma)=0 \Leftrightarrow \rho=\sigma . \\
D\left(\rho_{2}, \rho_{1}\right)+D\left(\rho_{3}, \rho_{2}\right) \geq D\left(\rho_{3}, \rho_{1}\right) .
\end{gathered}
$$

The last axiom is called the triangle inequality. In general, distances also satisfy the symmetric property:

$$
D(\rho, \sigma)=D(\sigma, \rho),
$$

but we do not require this property.

Complexity $C\left(\psi, \psi_{0}\right)$ shares the same properties (1)-(3):

$$
\begin{gathered}
C\left(\psi, \psi_{0}\right) \geq 0 . \\
C\left(\psi, \psi_{0}\right)=0 \Leftrightarrow|\psi\rangle=\left|\psi_{0}\right\rangle . \\
C\left(\psi_{2}, \psi_{1}\right)+C\left(\psi_{3}, \psi_{2}\right) \geq C\left(\psi_{3}, \psi_{1}\right) .
\end{gathered}
$$

The first and the second properties are trivial: The number of gates are non-negative, and if we do not use any gate, the final state is nothing but the initial state and vice versa. The triangle inequality Eq. (7) follows from the fact that the complexity $C\left(\psi, \psi^{\prime}\right)$ counts the minimum number of gates to reach $|\psi\rangle$ from $\left|\psi^{\prime}\right\rangle$. Thus, although it takes discrete values, gate complexity $C\left(\psi, \psi_{0}\right)$ is a sort of a distance

\footnotetext{
${ }^{3}$ If the two density matrices are pure states, they can be represented as $\rho=|\psi\rangle\langle\psi|$ and $\sigma=\left|\psi_{0}\right\rangle\left\langle\psi_{0}\right|$, for example.

${ }^{4}$ Relative entropy satisfies (1) and (2) but not (3) above. This can be checked easily for mixed states. For pure states, if two pure states are the same, then relative entropy becomes zero but for any different pure states, it diverges.
}

between $|\psi\rangle$ and $\left|\psi_{0}\right\rangle$ in the sense that it satisfies the axiom of the distance (5)-(7). ${ }^{5}$

In addition, holographic complexity takes continuous values; therefore, it is very natural to regard it as a distance shared with the properties of the gate complexity. However, under a few assumptions, we will show that there is no basis-independent distance dual to the holographic complexity, and thus conclude that holographic complexity should be a basis-dependent distance.

\section{BASIS INDEPENDENCE}

All of the physical observables are independent of the choices of the basis of the Hilbert space. Therefore, it seems to be natural that the quantum distance dual to holographic complexity is also basis independent. In addition to (1)-(3), the basis independence requires that distances satisfy

$$
D\left(U \rho U^{\dagger}, U \sigma U^{\dagger}\right)=D(\rho, \sigma),
$$

where $U$ is any unitary operator on the Hilbert space. In fact, well-known quantum distances, e.g., the trace distance and the quantum angle, ${ }^{6}$ satisfy this property [43].

However, this requirement of the basis independence imposes a very strong constraint on complexities as follows. Let us consider a distance with the basis independence (8) between two time-evolved states $|\psi(t)\rangle$ and $\left|\psi\left(t^{\prime}\right)\right\rangle$. Since Eq. (8) holds for any unitary operator $U=e^{-i H \delta t}$, the distance satisfies

$$
D\left(\psi(t), \psi\left(t^{\prime}\right)\right)=D\left(\psi\left(t-t^{\prime}\right), \psi(0)\right) ;
$$

i.e., the distance depends on just the difference of times. Thus, if the holographic complexity $C_{\mathrm{hol}}(t)$ is dual to such a distance, the triangle inequality

$D\left(\psi\left(t_{1}\right), \psi(0)\right)+D\left(\psi\left(t_{2}\right), \psi\left(t_{1}\right)\right) \geq D\left(\psi\left(t_{2}\right), \psi(0)\right)$

leads to the following inequality

$$
C_{\mathrm{hol}}\left(t_{1}\right)+C_{\mathrm{hol}}\left(t_{2}-t_{1}\right) \geq C_{\mathrm{hol}}\left(t_{2}\right),
$$

where $t_{2} \geq t_{1}$. We will see that this inequality does not match the known properties of the holographic complexity. Therefore, we should reject the requirement (8).

\footnotetext{
${ }^{5}$ Complexity can also satisfy the symmetric property $C\left(\psi, \psi_{0}\right)=$ $C\left(\psi_{0}, \psi\right)$ although we do not require it. Actually, if we choose the gate set so that it has all inverse gates of the gates in the set, the complexity satisfies the symmetric property.

${ }^{6}$ The trace distance is defined as $D^{\operatorname{tr}}(\rho, \sigma) \equiv \frac{1}{2} \operatorname{Tr} \sqrt{(\rho-\sigma)^{2}}$. The quantum angle is defined as $A(\rho, \sigma) \equiv \arccos F(\rho, \sigma)$, where $F(\rho, \sigma)$ is the fidelity $F(\rho, \sigma) \equiv \operatorname{Tr} \sqrt{\rho^{1 / 2} \sigma \rho^{1 / 2}}$. These $D^{\operatorname{tr}}(\rho, \sigma)$ and $A(\rho, \sigma)$ satisfy the axioms of distance (1)-(3) [43]. It is obvious from the definitions that they are basis independent.
} 


\section{LLOYD BOUND AND HOLOGRAPHIC COMPLEXITY}

A characteristic property of the holographic complexity is the saturation of the Lloyd bound [44]. Lloyd argued that the rate of computation by a physical device is limited by the energy; it is essentially due to the uncertainty principle

$$
\frac{1}{\Delta t} \sim M
$$

Based on this argument, it is conjectured that the growth rate of the holographic complexity is bounded by the mass $M$ of the black hole [6,7]:

$$
\frac{d C_{\mathrm{hol}}}{d t} \leq \alpha M
$$

where $\alpha$ is a numerical constant and its value is not important in the following discussion.

In $[6,7]$, it is also conjectured that the uncharged black holes saturate the bound (13) at late times

$$
\lim _{t \rightarrow \infty} \frac{d C_{\mathrm{hol}}}{d t}=\alpha M
$$

This is natural in the view that black holes are the fastest scrambler [45,46]; as far as complexity is associated with the growing black hole interiors at late times, its evolution is expected to take the maximum speed.

For example, in the CV conjecture with the definition of the holographic complexity as $C_{\text {hol }}(t)=\mathcal{V}(t) / G_{N} L$, where $\mathcal{V}(t)$ is the maximal volume ${ }^{7}$ at time $t$, and $G_{N}$ is Newton's constant and $L$ is the AdS radius, $C_{\text {hol }}(t)$ for an uncharged planar black hole satisfies

$$
\frac{d C_{\mathrm{hol}}}{d t} \leq \frac{16 \pi}{d-1} M
$$

for any $t[8],{ }^{8}$ and saturates the bound at late times [1,5].

On the other hand, in the CA conjecture, the bound (13) is violated although it behaves as (14) at late times [8]. See also [47] where the violation of the Lloyd bound for the holographic complexity is discussed. In any case, we next present the incompatibility between the inequality (11) and (13), (14).

\section{A NO-GO THEOREM}

We now show a no-go theorem that any basis-independent distance which satisfies the inequality (11) cannot be

\footnotetext{
${ }^{7}$ The maximal volume itself (and also the action on the WDW patch) is a UV-divergent quantity, and the UV-divergence is time independent [8]. We regularize the holographic complexity by subtracting that at $t=0$. Thus, $C_{\text {hol }}(t=0)=0$ in our regularization.

${ }^{8}$ The time $t$ is different from $t$ in [8] by a factor of two.
}

compatible with the Lloyd bound (13) and its saturation (14) at late times except for the case that the bound (13) is saturated for any time. Let us set $t_{1}=t$ and $t_{2}=2 t$ in the inequality (11). We then have the inequality

$$
2 C_{\text {hol }}(t) \geq C_{\text {hol }}(2 t) .
$$

If we define the following function $f(t)$,

$$
f(t) \equiv \alpha M t-C_{\mathrm{hol}}(t),
$$

then, from the inequality (16), it must satisfy

$$
f(2 t) \geq 2 f(t) .
$$

On the other hand, from the Lloyd bound (13), $f(t)$ clearly satisfies

$$
\frac{d f(t)}{d t} \geq 0 .
$$

We also have $f(t=0)=0$ since $C_{\mathrm{hol}}(t=0)=0$ from (6). With (19), this implies that $f(t)$ is a non-negative function;

$$
f \geq 0 \quad(\text { at } t \geq 0) .
$$

Furthermore, the saturation of the Lloyd bound at late times (14) implies that

$$
\lim _{t \rightarrow \infty} f(t)=\text { const } \equiv f_{0} \geq 0 .
$$

If we ignore the constraint (18) which comes from the triangle inequality with basis independence, there are infinite number of functions satisfying (19)-(21). However, these functions cannot satisfy the inequality (18) except for the case that the Lloyd bound is saturated at any time, i.e., $C_{\text {hol }}(t)=\alpha M t$ exactly for any time $t$ (or equivalently, $f(t) \equiv 0)$. This can be easily seen, since the triangle inequality (18) at late times implies

$$
\lim _{t \rightarrow \infty} 2 f(t) \leq \lim _{t \rightarrow \infty} f(2 t) \Leftrightarrow f_{0} \leq 0 .
$$

In other words, no function can satisfy (18) and (21) unless $f_{0}=0$ exactly. Here, $f_{0}=0$ is equivalent to

$$
\frac{d C_{\mathrm{hol}}}{d t}=\alpha M
$$

exactly for any time $t$ from (19) and (20).

On the other hand, we point out that there is no known bulk-defined holographic complexity which satisfies this property (23) for any time $t$, see [8].

Therefore, we conclude that holographic complexity, satisfying the late time behavior (14), cannot satisfy both the Lloyd bound (13) and the nature of the basis-independent distance (11). 


\section{ANOTHER ARGUMENT USING DIMENSIONAL ANALYSIS}

Here we provide another argument supporting our claim that basis-independent distances cannot be dual to holographic complexities, without relying on the Lloyd bound. The basis-independent distance might take the following form

$$
D(\rho, \sigma)=h(\operatorname{Tr} g(\rho, \sigma)),
$$

where $h$ is an arbitrary function $\mathbb{R} \rightarrow \mathbb{R}_{\geq 0}$, and $g$ is an arbitrary map from two density matrices to an operator on the Hilbert space. ${ }^{9}$ We concentrate on this class of distances in this section, although we are not sure if all basisindependent distances take the form (24). For this class of distances, the basis independence (8) is automatically satisfied, since they are defined with the trace. In addition, the distance is simplified for the case of two pure states $\rho=|\psi\rangle\langle\psi|$ and $\rho^{\prime}=\left|\psi^{\prime}\right\rangle\left\langle\psi^{\prime}\right|$. Actually, since pure states satisfy $\rho^{2}=\rho, \operatorname{Tr} \rho=1$, and also

$$
\operatorname{Tr}\left(\rho \rho^{\prime}\right)^{n}=\left|\left\langle\psi \mid \psi^{\prime}\right\rangle\right|^{2 n}
$$

then the distance can always be written as a function of the fidelity of the two pure states

$$
F\left(\psi, \psi^{\prime}\right) \equiv\left|\left\langle\psi \mid \psi^{\prime}\right\rangle\right|,
$$

which satisfies $0 \leq F\left(\psi, \psi^{\prime}\right) \leq 1$. (See also [48] for a discussion that a fidelity is related to the bulk volume.)

In literatures, holographic complexities are computed for eternal AdS black holes, which are dual to the thermofield double (TFD) states [49]. The TFD state is a pure state

$$
|\operatorname{TFD}(t=0)\rangle=\frac{1}{\sqrt{Z(\beta)}} \sum_{n} e^{-\beta E_{n} / 2}|n\rangle_{\mathrm{L}}|n\rangle_{\mathrm{R}}
$$

on the tensor product of the same two Hilbert spaces $\mathcal{H}_{\mathrm{L}} \otimes \mathcal{H}_{\mathrm{R}}$, where $Z(\beta) \equiv \sum_{n} e^{-\beta E_{n}}$ is the partition function on the single system at the inverse temperature $\beta$. Let us consider the time evolution of the TFD state by the total Hamiltonian $H_{\text {tot }}=H_{\mathrm{L}} \otimes \mathbf{1}_{\mathrm{R}}+\mathbf{1}_{\mathrm{L}} \otimes H_{\mathrm{R}}$, and consider the distance between the time-evolved states $|\operatorname{TFD}(t)\rangle$ and $|\operatorname{TFD}(t=0)\rangle$. Since they are pure states, the distance with the form (24) is a function of their fidelity. We represent the fidelity as $F(t)$, which takes a simple expression

$$
F(t) \equiv|\langle\operatorname{TFD}(t) \mid \operatorname{TFD}(t=0)\rangle|=\frac{|Z(\beta+2 i t)|}{Z(\beta)} .
$$

Here $Z(\beta+2 i t)$ means the analytic continuation, $\beta \rightarrow \beta+$ 2it, of the partition function $Z(\beta) .|Z(\beta+2 i t)|^{2}$ is called

\footnotetext{
${ }^{9} \mathrm{We}$ assume that $g(\rho, \sigma)$ can be expanded as series of $\rho$ and $\sigma$.
}

the spectral form factor [50,51], whose late time behavior is used to diagnose the discreteness of the black hole spectrum. Interestingly, since the fidelity $F(t)$ is nothing but the normalized spectral form factor, the distance of the TFD state at time $t$ from that at $t=0$ is a function of the spectral form factor. We note that the distance function (24) is introduced independently of states, so the functions $g$ and $h$ in (24) should be independent of the temperature. Thus, the temperature and time dependence in the distance between the time-evolved TFD states come only thorough the fidelity $F(t)$.

However, any function of the fidelity $F(t)$ cannot be the candidate for the holographic complexity, which can be seen as follows: At high temperature limit for any field theories on $d$-dimensional flat space, the leading parts of the partition functions take the following dimensionally determined form,

$$
Z(\beta) \sim \exp \left(c V \beta^{-d}\right)
$$

where $c$ is a constant which is roughly the number of degrees of freedom, and $V$ denotes the (regularized) spatial volume. ${ }^{10}$ Using Eq. (29), the fidelity $F(t)$ is given by

$F(t)=\exp \left[c V\left(\frac{-1}{\beta^{d}}+\frac{(\beta+2 i t)^{d}+(\beta-2 i t)^{d}}{2\left(\beta^{2}+4 t^{2}\right)^{d}}\right)\right]$.

At late times $t \gg \beta$, it is written as

$F(t) \sim \begin{cases}\exp \left[c V\left(\frac{-1}{\beta^{d}}+\frac{(-1)^{d / 2}}{(2 t)^{d}}\right)\right] & (d \text { : even }) \\ \exp \left[c V\left(\frac{-1}{\beta^{d}}+\frac{(-1)^{(d-1) / 2} d \beta}{(2 t)^{d+1}}\right)\right] & (d \text { : even })\end{cases}$

On the other hand, in the high temperature limit, the mass of the black hole is given by

$$
M \sim c V / \beta^{d+1},
$$

up to a numerical factor. Thus, the late time behavior of the holographic complexity, Eq. (14), is written as

$$
C_{\mathrm{hol}}(t) \sim c V t / \beta^{d+1} .
$$

Now it is clear that this (33) cannot be reproduced by any function of the fidelity (31) without using some other independent function of temperature. In other words, since the temperature and time dependence of any distance comes only through the fidelity, the holographic complexity is not dual to the class of distances (24). This implies

\footnotetext{
${ }^{10}$ This formula works even for theories on a curved space, as long as its curvature length scale is much bigger than the inverse of the temperature.
} 
that even though the spectral form factor (28) is useful to probe a structure of the spectrum and to diagnose the chaos [50,51], it fails to capture the fine-grained structure of the Hilbert space, necessary for complexity.

\section{DISCUSSIONS}

We have assumed that complexity can be defined as a distance in the Hilbert space like Nielsen's approach [37]. Then, if we further assume that the distance is basis independent, we have faced the mismatch with the holographic complexity. The assumption that complexity is a distance is probably reasonable because complexity should satisfy the same axioms of distance. The mismatch clearly comes from our assumption that the distance is basis independent (8).

Actually, the basis independence restricts the distances to the class as (24). For pure states $|\psi\rangle$ and $\left|\psi^{\prime}\right\rangle$, such a distance depends only on the absolute value of the inner product $\left|\left\langle\psi \mid \psi^{\prime}\right\rangle\right|$. As explained in [40], the inner product $\left|\left\langle\psi \mid \psi^{\prime}\right\rangle\right|$ loses much of the information of states; any states orthogonal to $|\psi\rangle$ are regarded as the most distant state from $|\psi\rangle$. Therefore, we cannot see the fine-grained structure of the system by this class of distances. ${ }^{11}$ However, holographic complexity needs to capture the fine-grained structure such that it keeps growing at late time.

Without the basis independence, we could not obtain the strong constraint from the triangle inequality (7). If we set $\psi_{1}=\psi(0), \psi_{2}=\psi(t)$ and $\psi_{3}=\psi(t+d t)$ in (7), we obtain

$$
C(t, 0)+C(t+d t, t) \geq C(t+d t, 0)
$$

which leads to the following inequality

$$
\frac{d C(t, 0)}{d t} \leq\left.\frac{d C\left(t^{\prime}, t\right)}{d t^{\prime}}\right|_{t^{\prime}=t}
$$

The inequality is a kind of the Lloyd bound. The growth rate of the complexity of $\psi(t)$ with the reference state $\psi(0)$ is bounded from the growth rate between two near states. Since this inequality holds between two different reference states, it doe not give a constraint to time dependence of complexities with the fixed reference state.

Our conclusion is that if holographic complexity is dual to a distance in the Hilbert space, it should be basis dependent. Since all physical observables in quantum mechanics are defined basis independently, this might sound a bit puzzling in the following sense: If we admit that holographic complexity is basis dependent, the

\footnotetext{
${ }^{11}$ Javier Magan and Henry Maxfield pointed out to us that basis independent distances saturate at $t=O(\beta)$; therefore, they cannot be a candidate of holographic complexity. We thank them for discussions of related issues.
}

growing volume of the wormhole cannot be observable. However, this is not a contradiction. To see this, remember that in holographic entanglement entropy [52], RT surface (or volume) is not observable since entanglement entropy is not directly observable.

In this paper, we have considered distances only between states at different times, and did not see the detailed dependence of the reference states. In literatures [32,33], unentangled states (or direct products states) are often taken as reference states. Here, "unentangle" means forming a direct product structure under the spatial (or geometrical) decomposition of the Hilbert space, and thus a specific basis respecting the spatial structure or locality is implicitly chosen. On the other hand, the basis-independent distances do not respect locality at all. Since basis independence Eq. (8) was required for arbitrary unitary transformations including highly nonlocal ones, this turns out too strong assumption. To seek for a good definition of complexity as a distance, we should respect the locality of quantum field theories. Representation of states by tensor networks, which gives a nice interpretation of growth of the wormhole [3], also suggests that we should respect the locality. If we perform the nonlocal transformations, such a local tensor structure of the state is lost. Furthermore, in order to define complexity, one needs to define, at first, a gate-set. If one changes the basis, the gate-set also changes and accordingly, the complexity (or distance in the Hilbert space) also changes for fixed target and reference states. ${ }^{12}$ This implies that there should exist a preferred choice of a gate-set which respects the locality of quantum field theories. In spin systems, to find such a locality-respecting gate-set is not that difficult. However, the real difficulty is for gauge theories where gauge constraint makes it complicated. Extended Hilbert space approaches are probably useful in such situations just as entanglement entropy case, see e.g., [53-56].

\section{ACKNOWLEDGMENTS}

Part of this work was presented by N. I. at the Yukawa Institute for Theoretical Physics (YITP) workshop, Holography, Quantum Entanglement and Higher Spin Gravity II, and "RIKEN-Osaka-OIST Joint Workshop 2018" at Okinawa Institute of Science and Technology (OIST). We would like to thank the audience for helpful feedback, especially Javier Magan and Henry Maxfield. We would also like to thank Keun-Young Kim for stimulating discussions and Javier Magan for useful comments on the manuscript. The work of K. H. was supported in part by JSPS KAKENHI Grants No. JP15H03658, No. JP15K13483, and No. JP17H06462. The work of N.I. was supported in part by JSPS KAKENHI Grant No. JP25800143. S. S. is supported in part by the Grantin-Aid for JSPS Research Fellow, Grant No. JP16J01004.

\footnotetext{
${ }^{12}$ If one changes the basis for states and gates in the same way, then the complexity is kept intact.
} 
[1] L. Susskind, Computational complexity and black hole horizons, Fortschr. Phys. 64, 24 (2016).

[2] L. Susskind, Addendum to computational complexity and black hole horizons, Fortschr. Phys. 64, 44 (2016).

[3] T. Hartman and J. Maldacena, Time evolution of entanglement entropy from black hole interiors, J. High Energy Phys. 05 (2013) 014.

[4] P. Hayden and J. Preskill, Black holes as mirrors: Quantum information in random subsystems, J. High Energy Phys. 09 (2007) 120.

[5] D. Stanford and L. Susskind, Complexity and shock wave geometries, Phys. Rev. D 90, 126007 (2014).

[6] A. R. Brown, D. A. Roberts, L. Susskind, B. Swingle, and Y. Zhao, Holographic Complexity Equals Bulk Action?, Phys. Rev. Lett. 116, 191301 (2016).

[7] A. R. Brown, D. A. Roberts, L. Susskind, B. Swingle, and Y. Zhao, Complexity, action, and black holes, Phys. Rev. D 93, 086006 (2016).

[8] D. Carmi, S. Chapman, H. Marrochio, R. C. Myers, and S. Sugishita, On the time dependence of holographic complexity, J. High Energy Phys. 11 (2017) 188.

[9] L. Susskind and Y. Zhao, Switchbacks and the bridge to nowhere, arXiv:1408.2823.

[10] O. Ben-Ami and D. Carmi, On volumes of subregions in holography and complexity, J. High Energy Phys. 11 (2016) 129.

[11] J. Couch, W. Fischler, and P. H. Nguyen, Noether charge, black hole volume, and complexity, J. High Energy Phys. 03 (2017) 119.

[12] S. Chapman, H. Marrochio, and R. C. Myers, Complexity of formation in holography, J. High Energy Phys. 01 (2017) 062.

[13] D. Carmi, R. C. Myers, and P. Rath, Comments on holographic complexity, J. High Energy Phys. 03 (2017) 118.

[14] M. Ghodrati, Complexity growth in massive gravity theories, the effects of chirality, and more, Phys. Rev. D 96, 106020 (2017).

[15] D. Carmi, More on holographic volumes, entanglement, and complexity, arXiv:1709.10463.

[16] J. Couch, S. Eccles, W. Fischler, and M. L. Xiao, Holographic complexity and noncommutative gauge theory, J. High Energy Phys. 03 (2018) 108.

[17] M. Moosa, Evolution of complexity following a global quench, J. High Energy Phys. 03 (2018) 031.

[18] A. P. Reynolds and S.F. Ross, Complexity of the AdS soliton, Classical Quantum Gravity 35, 095006 (2018).

[19] M. Moosa, Divergences in the rate of complexification, Phys. Rev. D 97, 106016 (2018).

[20] S. J. Zhang, Complexity and phase transitions in a holographic QCD model, Nucl. Phys. B929, 243 (2018).

[21] B. Swingle and Y. Wang, Holographic complexity of Einstein-Maxwell-dilaton gravity, arXiv:1712.09826.

[22] Z. Fu, A. Maloney, D. Marolf, H. Maxfield, and Z. Wang, Holographic complexity is nonlocal, J. High Energy Phys. 02 (2018) 072.

[23] Y. S. An and R. H. Peng, Effect of the dilaton on holographic complexity growth, Phys. Rev. D 97, 066022 (2018).

[24] M. Alishahiha, A. F. Astaneh, M. R. M. Mozaffar, and A. Mollabashi, Complexity growth with Lifshitz scaling and hyperscaling violation, J. High Energy Phys. 07 (2018) 042.
[25] B. Chen, W. M. Li, R. Q. Yang, C. Y. Zhang, and S. J. Zhang, Holographic subregion complexity under a thermal quench, J. High Energy Phys. 07 (2018) 034.

[26] S. Chapman, H. Marrochio, and R. C. Myers, Holographic complexity in Vaidya spacetimes I, J. High Energy Phys. 06 (2018) 046.

[27] R. Auzzi, S. Baiguera, and G. Nardelli, Volume and complexity for warped AdS black holes, J. High Energy Phys. 06 (2018) 063.

[28] P. Caputa, N. Kundu, M. Miyaji, T. Takayanagi, and K. Watanabe, Anti-de Sitter Space from Optimization of Path Integrals in Conformal Field Theories, Phys. Rev. Lett. 119, 071602 (2017).

[29] P. Caputa, N. Kundu, M. Miyaji, T. Takayanagi, and K. Watanabe, Liouville action as path-integral complexity: From continuous tensor networks to AdS/CFT, J. High Energy Phys. 11 (2017) 097.

[30] A. Bhattacharyya, P. Caputa, S. R. Das, N. Kundu, M. Miyaji, and T. Takayanagi, Path-integral complexity for perturbed CFTs, J. High Energy Phys. 07 (2018) 086.

[31] K. Hashimoto, N. Iizuka, and S. Sugishita, Time evolution of complexity in Abelian gauge theories, Phys. Rev. D 96, 126001 (2017).

[32] R. A. Jefferson and R. C. Myers, Circuit complexity in quantum field theory, J. High Energy Phys. 10 (2017) 107.

[33] S. Chapman, M. P. Heller, H. Marrochio, and F. Pastawski, Towards complexity for quantum field theory states, Phys. Rev. Lett. 120, 121602 (2018).

[34] L. Hackl and R. C. Myers, Circuit complexity for free fermions, arXiv:1803.10638.

[35] D. W. F. Alves and G. Camilo, Evolution of complexity following a quantum quench in free field theory, J. High Energy Phys. 06 (2018) 029.

[36] C. A. Agon, M. Headrick, and B. Swingle, Subsystem complexity and holography, arXiv:1804.01561.

[37] M. A. Nielsen, A geometric approach to quantum circuit lower bounds, arXiv:quant-ph/0502070.

[38] M. R. Dowling and Michael A. Nielsen, The geometry of quantum computation, arXiv:quant-ph/0701004.

[39] A. R. Brown, L. Susskind, and Y. Zhao, Quantum complexity and negative curvature, Phys. Rev. D 95, 045010 (2017).

[40] A. R. Brown and L. Susskind, The second law of quantum complexity, Phys. Rev. D 97, 086015 (2018).

[41] R.-Q. Yang, C. Niu, C.-Y. Zhang, K.-Y. Kim, Comparison of holographic and field theoretic complexities by time dependent thermofield double states, J. High Energy Phys. 02 (2018) 082.

[42] R. Khan, C. Krishnan, and S. Sharma, Circuit complexity in fermionic field theory, arXiv:1801.07620.

[43] M. A. Nielsen and I. L. Chuang, Quantum Computation and Quantum Information (Cambridge University Press, Cambridge, England, 2000).

[44] S. Lloyd, Ultimate physical limits to computation, Nature (London) 406, 1047 (2000).

[45] Y. Sekino and L. Susskind, Fast scramblers, J. High Energy Phys. 10 (2008) 065.

[46] J. Maldacena, S. H. Shenker, and D. Stanford, A bound on chaos, J. High Energy Phys. 08 (2016) 106. 
[47] W. Cottrell and M. Montero, Complexity is simple!, J. High Energy Phys. 02 (2018) 039.

[48] M. Miyaji, T. Numasawa, N. Shiba, T. Takayanagi, and K. Watanabe, Distance between Quantum States and Gauge-Gravity Duality, Phys. Rev. Lett. 115, 261602 (2015).

[49] J. M. Maldacena, Eternal black holes in anti-de Sitter, J. High Energy Phys. 04 (2003) 021.

[50] E. Dyer and G. Gur-Ari, 2D CFT partition functions at late times, J. High Energy Phys. 08 (2017) 075.

[51] J. S. Cotler, G. Gur-Ari, M. Hanada, J. Polchinski, P. Saad, S. H. Shenker, D. Stanford, A. Streicher, and M. Tezuka, Black holes and random matrices, J. High Energy Phys. 05 (2017) 118.
[52] S. Ryu and T. Takayanagi, Holographic Derivation of Entanglement Entropy from AdS/CFT, Phys. Rev. Lett. 96, 181602 (2006).

[53] S. Ghosh, R. M. Soni, and S. P. Trivedi, On the entanglement entropy for gauge theories, J. High Energy Phys. 09 (2015) 069.

[54] R. M. Soni and S. P. Trivedi, Aspects of entanglement entropy for gauge theories, J. High Energy Phys. 01 (2016) 136.

[55] S. Aoki, T. Iritani, M. Nozaki, T. Numasawa, N. Shiba, and H. Tasaki, On the definition of entanglement entropy in lattice gauge theories, J. High Energy Phys. 06 (2015) 187.

[56] S. Aoki, N. Iizuka, K. Tamaoka, and T. Yokoya, Entanglement entropy for 2D gauge theories with matters, Phys. Rev. D 96, 045020 (2017). 